\title{
ANALYSIS OF POSITIVE LINEAR CONTINUOUS-TIME SYSTEMS USING THE CONFORMABLE DERIVATIVE
}

\author{
TADEUSZ KACZOREK ${ }^{a}$ \\ ${ }^{a}$ Faculty of Electrical Engineering \\ Białystok Technical University, Wiejska 45D, 15-351 Białystok, Poland \\ e-mail: kaczorek@ee.pw.edu.pl
}

\begin{abstract}
Positive linear continuous-time systems are analyzed via conformable fractional calculus. A solution to a fractional linear system is derived. Necessary and sufficient conditions for the positivity of linear systems are established. Necessary and sufficient conditions for the asymptotic stability of positive linear systems are also given. The solutions of positive fractional linear systems based on the Caputo and conformable definitions are compared.
\end{abstract}

Keywords: conformable fractional derivative, positive linear system, stability.

\section{Introduction}

A dynamical system is called positive if its trajectory starting from any nonnegative initial state remains forever in the positive orthant for all nonnegative inputs. An overview of state of the art in positive theory is given in the monographs by Farina and Rinaldi (2000) as well as Kaczorek (2002). A variety of models having positive behavior can be found in engineering, especially in electrical circuits (Kaczorek and Rogowski, 2015), economics, social sciences, biology and medicine, etc. (Farina and Rinaldi, 2000; Kaczorek, 2002).

Positive electrical circuits were analyzed by Kaczorek (2015a; 2013a; 2014; 2016a; 2015b; 2011a; 2010). The constructability and observability of standard and positive electrical circuits were addressed also by Kaczorek (2013a), along with the decoupling zeros (Kaczorek, 2014) and minimal-phase positive electrical circuits (Kaczorek, 2016a). A new class of normal positive linear electrical circuits was introduced by Kaczorek (2015b), who also investigated positive fractional linear electrical circuits (Kaczorek, 2013b), positive linear systems with different fractional orders (Kaczorek, 2010; 2011b) and positive unstable electrical circuits (Kaczorek, 2012). The zeroing of state variables in descriptor electrical circuits was addressed by Kaczorek (2013c), while the realization problem of positive linear systems by Benvenuti and Farina (2004). Positive electrical circuits with zero transfer matrices were analyzed by Kaczorek (2016b).

In the work of Khalil et al. (2014) a new definition of the fractional derivative called "conformable" was proposed, and in that of Abdeljawad (2015) fundamentals of the conformable fractional calculus were developed. In this paper, positive linear continuous-time systems will be analyzed with the use of the conformable derivative.

The paper is organized as follows. In Section 2 some preliminaries concerning the conformable fractional calculus are presented. The positive fractional continuous-time linear systems are addressed in Section 3. The stability of the positive fractional linear systems is analyzed in Section 4. Comparison of the solutions of fractional linear systems using the Caputo and conformable definitions is presented in Section 5. Concluding remarks are given in Section 6.

The following notation will be used: $\mathbb{R}$, the set of real numbers; $\mathbb{R}^{n \times m}$, the set of $n \times m$ real matrices; $\mathbb{R}_{+}^{n \times m}$, the set of $n \times m$ real matrices with nonnegative entries and $\mathbb{R}_{+}^{n}=\mathbb{R}_{+}^{n \times 1} ; M_{n}$, the set of $n \times n$ Metzler matrices (real matrices with nonnegative off-diagonal entries); $I_{n}$, the $n \times n$ identity matrix; $A^{T}$ denotes the transpose of matrix $A$.

\section{Preliminaries}

The following conformable derivative of the function $f(t)$ of order $\alpha, 0<\alpha<1$ will be used (Khalil et al., 2014): 


$$
\frac{\mathrm{d}^{\alpha} f(t)}{\mathrm{d} t^{\alpha}}=\lim _{\varepsilon \rightarrow 0} \frac{f\left(t+\varepsilon t^{1-\alpha}\right)-f(t)}{\varepsilon}, \quad t>0 .
$$

If (11) exists then the function $f(t)$ is called $\alpha$-differentiable (Khalil et al., 2014).

Theorem 1. (Khalil et al., 2014) If the functions $f(t)$ and $g(t)$ are $\alpha$-differentiable, $0<\alpha<1$ then

$$
\frac{\mathrm{d}^{\alpha}}{\mathrm{d} t^{\alpha}}[a f(t)+b y(t)]=a \frac{\mathrm{d}^{\alpha} f(t)}{\mathrm{d} t^{\alpha}}+b \frac{\mathrm{d}^{\alpha} g(t)}{\mathrm{d} t^{\alpha}},
$$

where $a, b \in \mathbb{R}$ and

$$
\begin{gathered}
\frac{\mathrm{d}^{\alpha}}{\mathrm{d} t^{\alpha}}[f(t) g(t)]=f(t) \frac{\mathrm{d}^{\alpha} g(t)}{\mathrm{d} t^{\alpha}}+g(t) \frac{\mathrm{d}^{\alpha} f(t)}{\mathrm{d} t^{\alpha}}, \\
\frac{\mathrm{d}^{\alpha}}{\mathrm{d} t^{\alpha}}\left[\frac{f(t)}{g(t)}\right]=\frac{g(t) \frac{\mathrm{d}^{\alpha} f(t)}{\mathrm{d} t^{\alpha}}-f(t) \frac{\mathrm{d}^{\alpha} g(t)}{\mathrm{d} t^{\alpha}}}{[g(t)]^{2}}, \\
\frac{\mathrm{d}^{\alpha} f(t)}{\mathrm{d} t^{\alpha}}=t^{1-\alpha} \frac{\mathrm{d} f(t)}{\mathrm{d} t} \\
\frac{\mathrm{d}^{\alpha} t^{q}}{\mathrm{~d} t^{\alpha}}=q t^{q-\alpha} \quad \text { for all } q \in \mathbb{R}, \\
\frac{\mathrm{d}^{\alpha} e^{q t}}{\mathrm{~d} t^{\alpha}}=q t^{1-\alpha} e^{q t} \quad \text { for all } q \in \mathbb{R} .
\end{gathered}
$$

\section{Positive fractional continuous-time linear systems}

Consider the fractional continuous-time linear system described by the equations

$$
\begin{aligned}
\frac{\mathrm{d}^{\alpha} x(t)}{\mathrm{d} t^{\alpha}} & =A x(t)+B u(t), \quad 0<\alpha<1, \\
y(t) & =C x(t)+D u(t),
\end{aligned}
$$

where $x(t) \in \mathbb{R}^{n}, u(t) \in \mathbb{R}^{m}, y(t) \in \mathbb{R}^{p}$ are the state, input and output vectors, respectively, and $A \in \mathbb{R}^{n \times n}$, $B \in \mathbb{R}^{n \times m}, C \in \mathbb{R}^{p \times n}, D \in \mathbb{R}^{p \times m}$.

Theorem 2. The solution $x(t)$ of the equation (8a) has the form

$$
\begin{aligned}
& x(t)=e^{\frac{A}{\alpha} t^{\alpha}} x_{0}+\int_{0}^{t} e^{\frac{A}{\alpha}\left(t^{\alpha}-\tau^{\alpha}\right)} B u(\tau) \tau^{\alpha-1} \mathrm{~d} \tau, \\
& x(0)=x_{0},
\end{aligned}
$$

where

$$
e^{\frac{A}{\alpha} t^{\alpha}}=\sum_{k=0}^{\infty} \frac{A^{k} t^{k \alpha}}{\alpha^{k} k !}
$$

Proof. It will be shown that the solution (9) satisfies Eqn. (8a). Using (5)-(7), it is easy to check that $x(0)=x_{0}$ and

$$
\begin{aligned}
\frac{\mathrm{d}^{\alpha} x(t)}{\mathrm{d} t^{\alpha}}= & \frac{\mathrm{d}^{\alpha}}{\mathrm{d} t^{\alpha}}\left[e^{\frac{A}{\alpha} t^{\alpha}} x_{0}\right. \\
& \left.+\int_{0}^{t} e^{\frac{A}{\alpha}\left(t^{\alpha}-\tau^{\alpha}\right)} B u(\tau) \tau^{\alpha-1} \mathrm{~d} \tau\right] \\
= & A\left[e^{\frac{A}{\alpha} t^{\alpha}} x_{0}+\int_{0}^{t} e^{\frac{A}{\alpha}\left(t^{\alpha}-\tau^{\alpha}\right)} B u(\tau) \tau^{\alpha-1} \mathrm{~d} \tau\right] \\
& +B u(t) \\
= & A x(t)+B u(t) .
\end{aligned}
$$

In the work of Abdeljawad (2015) the proof is based on the Laplace transform.

Definition 1. The fractional linear system (8) is called (internally) positive if $x(t) \in \mathbb{R}_{+}^{n}, y(t) \in \mathbb{R}_{+}^{p}$ for all $x_{0} \in$ $\mathbb{R}_{+}^{n}$ and any $u(t) \in \mathbb{R}_{+}^{m}, t \geq 0$.

Definition 2. A matrix $A=\left[a_{i j}\right] \in \mathbb{R}^{n \times n}$ is called the Metzler matrix if $a_{i j} \geq 0$ for $i \neq j$ and $i, j=1, \ldots, n$.

Theorem 3. The fractional linear system (8) is (internally) positive if and only if

$$
A \in M_{n}, \quad B \in \mathbb{R}_{+}^{n \times m}, \quad C \in \mathbb{R}_{+}^{p \times n}, \quad D \in \mathbb{R}_{+}^{p \times m} .
$$

Proof. First we shall show that

$$
e^{\frac{A}{\alpha} t^{\alpha}} \in \mathbb{R}_{+}^{n \times n} \text { for } t \geq 0,0<\alpha<1
$$

if and only if $A \in M_{n}$.

From (10) written in the form

$$
e^{\frac{A}{\alpha} t^{\alpha}}=I_{n}+\frac{A t^{\alpha}}{\alpha}+\ldots
$$

it follows that $e^{\frac{A}{\alpha} t^{\alpha}} \in \mathbb{R}_{+}^{n \times n}$ for small $t>0$ only if $A \in$ $M_{n}$.

If $A / \alpha \in M_{n}$ then there exists $\lambda>0$ such that $A / \alpha+I_{n} \lambda \in \mathbb{R}_{+}^{n \times n}$ and

$$
e^{\frac{A}{\alpha} t^{\alpha}}=e^{\left(\frac{A}{\alpha}+I_{n} \lambda\right) t^{\alpha}} e^{-\lambda I_{n} t^{\alpha}} \in \mathbb{R}_{+}^{n \times n}, \quad t \geq 0
$$

since $e^{\left(\frac{A}{\alpha}+I_{n} \lambda\right) t^{\alpha}} \in \mathbb{R}_{+}^{n \times n}$ and $e^{-\lambda I_{n} t^{\alpha}} \in \mathbb{R}_{+}^{n \times n}, t \geq 0$.

If $A \in M_{n}, B \in \mathbb{R}_{+}^{n \times m}, x_{0} \in \mathbb{R}_{+}^{n}$ and $u(t) \in \mathbb{R}_{+}^{m}$, $t \geq 0$ then

$$
e^{\frac{A}{\alpha} t^{\alpha}} x_{0} \in \mathbb{R}_{+}^{n}, \quad e^{\frac{A}{\alpha} t^{\alpha}} B u(t) \in \mathbb{R}_{+}^{n} \quad \text { for } t \geq 0
$$

and from (9) we obtain $x(t) \in \mathbb{R}_{+}^{n}$ for $t \geq 0$.

If $x(t) \in \mathbb{R}_{+}^{n}$ and $u(t) \in \mathbb{R}_{+}^{m}, t \geq 0$ then from (8b) we have $y(t) \in \mathbb{R}_{+}^{p}, t \geq 0$. 
To show the necessity, we assume that $u(t)=0, t \geq$ 0 and $x_{0}=e_{i}$ (the $i$-th column of the matrix $I_{n}$ ). The trajectory $x(t)$ does not leave orthant $\mathbb{R}_{+}^{n}$ only if

$$
\left.\frac{\mathrm{d}^{\alpha} x(t)}{\mathrm{d} t^{\alpha}}\right|_{t=0}=A e_{i} \in \mathbb{R}_{+}^{n},
$$

which implies $a_{i j} \geq 0$ for $i \neq j$. Therefore, we have $A \in M_{n}$.

For the same reason, for $x_{0}=0$ we have

$$
\left.\frac{\mathrm{d}^{\alpha} x(t)}{\mathrm{d} t^{\alpha}}\right|_{t=0}=B u(0) \in \mathbb{R}_{+}^{n},
$$

what implies $B \in \mathbb{R}_{+}^{n \times m}$ since $u(0) \in \mathbb{R}_{+}^{m}$ can be arbitrary. From (8b) for $u(t)=0, t \geq 0$ we have $y(0)=C x_{0} \in \mathbb{R}_{+}^{p}$ and $C \in \mathbb{R}_{+}^{p \times n}$ since $x_{0} \in \mathbb{R}_{+}^{n}$ can be arbitrary. In a similar way, we have $y(0)=D u(0) \in \mathbb{R}_{+}^{p}$ and $D \in \mathbb{R}_{+}^{p \times m}$ since $u(0) \in \mathbb{R}_{+}^{m}$ can be arbitrary.

\section{Stability of positive fractional linear systems}

Consider the positive fractional continuous-time linear system

$$
\frac{\mathrm{d}^{\alpha} x(t)}{\mathrm{d} t^{\alpha}}=A x(t), \quad x(0)=x_{0} \in \mathbb{R}_{+}^{n},
$$

where $x(t) \in \mathbb{R}_{+}^{n}, t \geq 0, A \in M_{n}$.

Definition 3. (Kaczorek, 2011; Kaczorek and Rogowski, 2015) The positive fractional system (17) is called asymptotically stable if

$$
\lim _{t \rightarrow \infty} x(t)=0 \quad \text { for any } x_{0} \in \mathbb{R}_{+}^{n} .
$$

Theorem 4. The positive fractional system (17) is asymptotically stable if and only if one of the following equivalent conditions are satisfied:

1. There exists a strictly positive vector $\lambda^{T}=$ $\left[\begin{array}{ccc}\lambda_{1} & \cdots & \lambda_{n}\end{array}\right], \lambda_{k}>0, k=1, \ldots, n$ such that

$$
A \lambda<0 \text {. }
$$

2. The coefficients of the characteristic polynomial of the matrix $A$

$$
\begin{aligned}
& \operatorname{det}\left[I_{n} s-A\right] \\
& \quad=s^{n}+a_{n-1} s^{n-1}+\cdots+a_{1} s+a_{0}
\end{aligned}
$$

are positive, i.e., $a_{k}>0$ for $k=0,1, \ldots, n-1$.

3. The principal minors of the matrix

$$
\bar{A}=-A=\left[\begin{array}{ccc}
\bar{a}_{11} & \cdots & \bar{a}_{1 n} \\
\vdots & \ddots & \vdots \\
\bar{a}_{n 1} & \cdots & \bar{a}_{n n}
\end{array}\right]
$$

are positive, i.e.,

$$
\begin{gathered}
\bar{a}_{11}>0, \quad\left[\begin{array}{cc}
\bar{a}_{11} & \bar{a}_{12} \\
\bar{a}_{21} & \bar{a}_{22}
\end{array}\right]>0, \\
\ldots, \quad \operatorname{det}[-A]>0 .
\end{gathered}
$$

Proof. Let $I_{\alpha}(0, t)$ be the fractional integral operator of order $\alpha, 0<\alpha<1$, satisfying (Abdeljawad, 2015)

$$
I_{\alpha}(0, t) \frac{\mathrm{d}^{\alpha} x(t)}{\mathrm{d} t^{\alpha}}=x(t)-x(0) .
$$

Applying this operator to (17), for $\lim _{t \rightarrow \infty} x(t)=0$ and $x(0)>0$ we obtain

$$
-x(0)=A I_{\alpha}(0, \infty)=A \lambda<0
$$

since the fractional integral of $x(t)$ belongs to $\mathbb{R}_{+}^{n}$ and $I_{\alpha}(0, \infty)>0$.

Note that the dual system

$$
\frac{\mathrm{d}^{\alpha} x(t)}{\mathrm{d} t^{\alpha}}=A^{T} x(t)
$$

is asymptotically stable if and only if the system 177 is asymptotically stable.

As a Lyapunov function of the dual system we choose

$$
V(x)=x^{T}(t) \lambda>0 \quad \text { for } x(t)>0
$$

and, using (25), we obtain

$$
\frac{\mathrm{d}^{\alpha} V(x)}{\mathrm{d} t^{\alpha}}=\frac{\mathrm{d}^{\alpha} x^{T}(t)}{\mathrm{d} t^{\alpha}} \lambda=x^{T}(t) A \lambda<0
$$

if the condition (19) is satisfied. Therefore, the positive fractional system (17) is asymptotically stable if and only if Condition 1 is satisfied.

The equivalence of Conditions 1 and 2, as well as 2 and 3, has been shown by Kaczorek (2011c) and Kaczorek and Rogowski (2015).

Theorem 5. The positive fractional system (17) is unstable if at least one diagonal entry of the matrix $A \in M_{n}$ is nonnegative.

Proof. Let all entries of the $i$-th row of the matrix $A$ be nonnegative. Then there is no strictly positive vector $\lambda \in \mathbb{R}_{+}^{n}$ such that (19) holds. Therefore, by Condition 1 of Theorem 4 the system is unstable.

Remark 1. If $\operatorname{det} A \neq 0$ then as the vector $\lambda$ we may choose

$$
\lambda=A^{-1} c \quad \text { for any } c<0, c \in \mathbb{R}^{n}
$$

since $A \lambda=A A^{-1} c<0$. 
Example 1. Consider the positive fractional system (17) with

$$
A=\left[\begin{array}{ccc}
-2 & 1 & 0 \\
1 & -3 & 1 \\
1 & 0 & -2
\end{array}\right] \in M_{3}
$$

The system is asymptotically stable since for $\lambda^{T}=$ [ $\left.\begin{array}{lll}1 & 1 & 1\end{array}\right]$ we have

$$
A \lambda=\left[\begin{array}{ccc}
-2 & 1 & 0 \\
1 & -3 & 1 \\
1 & 0 & -2
\end{array}\right]\left[\begin{array}{l}
1 \\
1 \\
1
\end{array}\right]=\left[\begin{array}{l}
-1 \\
-1 \\
-1
\end{array}\right]<0 \text {. }
$$

Using (28) and (29), we obtain

$$
\begin{aligned}
\lambda & =A^{-1} c \\
& =\left[\begin{array}{ccc}
-2 & 1 & 0 \\
1 & -3 & 1 \\
1 & 0 & -2
\end{array}\right]^{-1}\left[\begin{array}{l}
c_{1} \\
c_{2} \\
c_{3}
\end{array}\right] \\
& =-\frac{1}{9}\left[\begin{array}{c}
6 c_{1}+2 c_{2}+c_{3} \\
3 c_{1}+4 c_{2}+2 c_{3} \\
3 c_{1}+c_{2}+5 c_{3}
\end{array}\right]<0
\end{aligned}
$$

for any nonzero $c \in \mathbb{R}_{+}^{3}$.

The characteristic polynomial of 29]

$$
\begin{aligned}
\operatorname{det}\left[I_{3} s-A\right] & =\left[\begin{array}{ccc}
s+2 & -1 & 0 \\
-1 & s+3 & -1 \\
-1 & 0 & s+2
\end{array}\right] \\
& =s^{3}+7 s^{2}+15 s+9
\end{aligned}
$$

has all positive coefficients and its zeros are $s_{1}=-1$, $s_{2}=s_{3}=-3$.

Condition 2 of Theorem 4 is satisfied and the system is asymptotically stable. Condition 3 of the same theorem is also satisfied since the principal minors of the matrix

$$
\bar{A}=-A=\left[\begin{array}{ccc}
2 & -1 & 0 \\
-1 & 3 & -1 \\
-1 & 0 & 2
\end{array}\right]
$$

are positive, i.e.,

$$
\begin{gathered}
\bar{a}_{11}=2 \\
\left|\begin{array}{ll}
\bar{a}_{11} & \bar{a}_{12} \\
\bar{a}_{21} & \bar{a}_{22}
\end{array}\right|=\left|\begin{array}{cc}
2 & -1 \\
-1 & 3
\end{array}\right|=5, \\
\operatorname{det} \bar{A}=\left|\begin{array}{ccc}
2 & -1 & 0 \\
-1 & 3 & -1 \\
-1 & 0 & 2
\end{array}\right|=9 .
\end{gathered}
$$

Comparing the asymptotic stability conditions for the Caputo and conformable definitions, we obtain the following corollary.

Corollary 1. The asymptotic stability conditions for positive continuous-time linear systems for the Caputo and conformable definitions of the fractional derivative are the same.

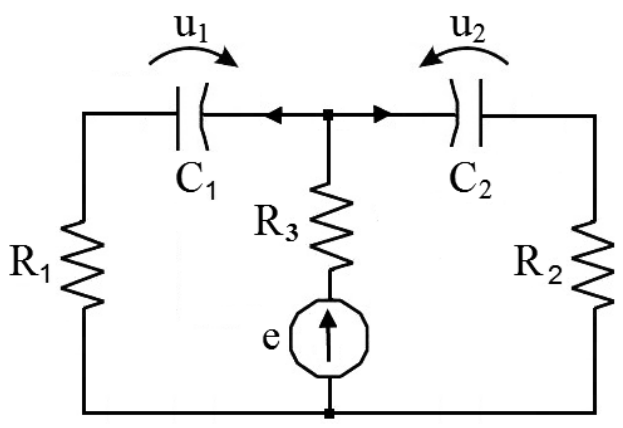

Fig. 1. Electrical circuit of Example 2.

\section{Comparison of solutions of fractional linear continuous-time systems based on the Caputo and conformable derivative definitions}

In this section the solutions of the positive fractional linear electrical circuits based on the Caputo and conformable derivative definitions will be presented and compared.

Example 2. Consider the electrical circuit shown in Fig. 1 with given resistances $R_{1}, R_{2}, R_{3}$, capacitances $C_{1}, C_{2}$ and source voltage $e$.

Using Kirchhoff's laws, we may write the equations

$$
\begin{aligned}
& e=R_{1} C_{1} \frac{\mathrm{d}^{\alpha} u_{1}}{\mathrm{~d} t^{\alpha}}+u_{1}+R_{3}\left(C_{1} \frac{\mathrm{d}^{\alpha} u_{1}}{\mathrm{~d} t^{\alpha}}+C_{2} \frac{\mathrm{d}^{\alpha} u_{2}}{\mathrm{~d} t^{\alpha}}\right), \\
& e=R_{3}\left(C_{1} \frac{\mathrm{d}^{\alpha} u_{1}}{\mathrm{~d} t^{\alpha}}+C_{2} \frac{\mathrm{d}^{\alpha} u_{2}}{\mathrm{~d} t^{\alpha}}\right)+u_{2}+R_{2} C_{2} \frac{\mathrm{d}^{\alpha} u_{2}}{\mathrm{~d} t^{\alpha}},
\end{aligned}
$$

which can be put down in the form

$$
\begin{gathered}
{\left[\begin{array}{cc}
\left(R_{1}+R_{3}\right) C_{1} & R_{3} C_{2} \\
R_{3} C_{1} & \left(R_{2}+R_{3}\right) C_{2}
\end{array}\right] \frac{\mathrm{d}^{\alpha}}{\mathrm{d} t^{\alpha}}\left[\begin{array}{l}
u_{1} \\
u_{2}
\end{array}\right]} \\
=\left[\begin{array}{cc}
-1 & 0 \\
0 & -1
\end{array}\right]\left[\begin{array}{l}
u_{1} \\
u_{2}
\end{array}\right]+\left[\begin{array}{l}
1 \\
1
\end{array}\right] e .
\end{gathered}
$$

Premultiplying (35) by the inverse matrix

$$
\begin{aligned}
& {\left[\begin{array}{cc}
\left(R_{1}+R_{3}\right) C_{1} & R_{3} C_{2} \\
R_{3} C_{1} & \left(R_{2}+R_{3}\right) C_{2}
\end{array}\right]^{-1}} \\
& \quad=\frac{1}{\Delta}\left[\begin{array}{cc}
\left(R_{2}+R_{3}\right) C_{2} & -R_{3} C_{2} \\
-R_{3} C_{1} & \left(R_{1}+R_{3}\right) C_{1}
\end{array}\right],
\end{aligned}
$$

where

$$
\Delta=\left[R_{1}\left(R_{2}+R_{3}\right)+R_{2} R_{3}\right] C_{1} C_{2}
$$

we obtain

$$
\frac{\mathrm{d}^{\alpha}}{\mathrm{d} t^{\alpha}}\left[\begin{array}{l}
u_{1} \\
u_{2}
\end{array}\right]=A\left[\begin{array}{l}
u_{1} \\
u_{2}
\end{array}\right]+B e,
$$


where

$$
\begin{aligned}
A & =\frac{1}{\Delta}\left[\begin{array}{cc}
-\left(R_{2}+R_{3}\right) C_{2} & R_{3} C_{2} \\
R_{3} C_{1} & -\left(R_{1}+R_{3}\right) C_{1}
\end{array}\right], \\
B & =\frac{1}{\Delta}\left[\begin{array}{l}
R_{2} C_{2} \\
R_{1} C_{1}
\end{array}\right] .
\end{aligned}
$$

From (37b) it follows that the electrical circuit is positive since $A \in M_{2}$ and $B$ has positive entries.

For further analysis we assume $\alpha=0.5, R_{1}=R_{2}=$ $10 \Omega, R_{3}=20 \Omega, C_{1}=C_{2}=100 \mathrm{mF}$ and the constant input $e=1 \mathrm{~V}$. It is assumed that the initial conditions $x(0)=0$ and $\left[u_{1}(0) \quad u_{2}(0)\right]^{T}=0$.

Using the Caputo definition of the fractional derivative (Kaczorek, 2011c), we obtain the following solution to (37):

$$
\begin{aligned}
x(t) & =\int_{0}^{t} \Phi(t-\tau) B \mathrm{~d} \tau \\
& =\sum_{k=0}^{\infty}\left(A^{k} \int_{0}^{t} \frac{(t-\tau)^{(k+1) \alpha-1}}{\Gamma[(k+1) \alpha]} \mathrm{d} \tau\right) B \\
& =\sum_{k=0}^{\infty} \frac{A^{k} t^{(k+1) \alpha}}{\Gamma[(k+1) \alpha+1]} B \\
& =\sum_{k=0}^{\infty} \frac{t^{0.5(k+1)}}{\Gamma[0.5 k+1.5]}\left[\begin{array}{cc}
-0.6 & 0.4 \\
0.4 & -0.6
\end{array}\right]^{k}\left[\begin{array}{l}
0.2 \\
0.2
\end{array}\right]
\end{aligned}
$$

and, using (9),

$$
\begin{aligned}
x(t) & =\int_{0}^{t} e^{\frac{A}{\alpha}\left(t^{\alpha}-\tau^{\alpha}\right)} B \tau^{\alpha-1} \mathrm{~d} \tau \\
& =e^{\frac{A}{\alpha} t^{\alpha}} \int_{0}^{t} e^{-\frac{A}{\alpha} \tau^{\alpha}} \tau^{\alpha-1} \mathrm{~d} \tau B \\
& =\sum_{k=0}^{\infty} \frac{A^{k} t^{k \alpha}}{\alpha^{k} k !}\left(\int_{0}^{t} \sum_{k=0}^{\infty} \frac{(-A)^{k} \tau^{k \alpha}}{\alpha^{k} k !} \tau^{\alpha-1} \mathrm{~d} \tau\right) B \\
& =\sum_{k=0}^{\infty} \frac{A^{k} t^{k \alpha}}{\alpha^{k} k !}\left(\sum_{k=0}^{\infty} \frac{(-A)^{k}}{\alpha^{k} k !} \int_{0}^{t} \tau^{(k+1) \alpha-1} \mathrm{~d} \tau\right) B \\
& =\sum_{k=0}^{\infty} \frac{A^{k} t^{k \alpha}}{\alpha^{k} k !}\left(\sum_{k=0}^{\infty} \frac{(-A)^{k} t^{(k+1) \alpha}}{\alpha^{k} k ![(k+1) \alpha]}\right) B \\
= & \sum_{k=0}^{\infty} \frac{t^{0.5 k}}{0.5^{k} k !}\left[\begin{array}{cc}
0.4 & -0.6
\end{array}\right]^{k} \\
& \times\left(\sum_{k=0}^{\infty} \frac{t^{0.5(k+1)}}{0.5^{k} k ![0.5 k+0.5]}\left[\begin{array}{cc}
0.6 & -0.4 \\
-0.4 & 0.6
\end{array}\right]^{k}\right) \\
& \times\left[\begin{array}{ll}
0.2 \\
0.2
\end{array}\right]
\end{aligned}
$$

From Fig. 2 it follows that for the conformable definition the electrical circuit reaches its steady state in a shorter time.

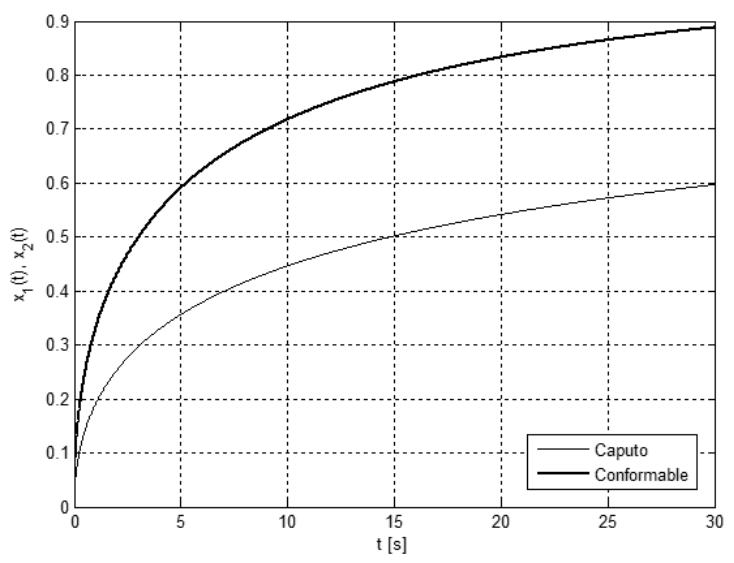

Fig. 2. Comparison of the solutions (38) and 39].

\section{Concluding remarks}

The positive linear continuous-time systems have been analyzed with the use of the conformable derivative. Solutions to fractional linear systems has been derived (Theorem 2). Necessary and sufficient conditions for the positivity of the fractional linear systems have been established (Theorem 3). Necessary and sufficient conditions for the asymptotic stability of the positive linear systems have been given (Theorem 4).

The solutions of the positive fractional linear systems based on the Caputo and conformable definitions have been presented and compared. The discussion has been illustrated via an example of a positive electrical circuit.

The findings can be extended to linear systems with delays.

\section{Acknowledgment}

This work was supported by the National Science Centre in Poland under the grant no. 2014/13/B/ST7/03467.

\section{References}

Abdeljawad, T. (2015). On conformable fractional calculus, Journal of Computational and Applied Mathematics 279: 57-66.

Benvenuti, L. and Farina, L. (2004). A tutorial on the positive realization problem, IEEE Transactions on Automatic Control 49(5): 651-664.

Farina, L. and Rinaldi, S. (2000). Positive Linear Systems, Theory and Applications, Wiley, New York, NY.

Kaczorek, T. (2016a). Minimal-phase positive electrical circuits, Electrical Review 92(3): 182-189.

Kaczorek, T. (2016b). Positive electrical circuits with zero transfer matrices and their discretization, Computer Applications in Electrical Engineering 14: 1-13.

Kaczorek, T. (2015a). A class of positive and stable time-varying electrical circuits, Electrical Review 91(5): 121-124. 
Kaczorek, T. (2015b). Normal positive electrical circuits, IET Circuits Theory and Applications 9(5): 691-699.

Kaczorek, T. (2014). Decoupling zeros of positive continuous-time linear systems and electrical circuits, in J. Świątek et al. (Eds.), Advances in Systems Science, Springer, Cham, pp. 1-15.

Kaczorek, T. (2013a). Constructability and observability of standard and positive electrical circuits, Electrical Review 89(7): 132-136.

Kaczorek, T. (2013b). Positive fractional linear electrical circuits, Proceedings of SPIE 8903, Art. No. 3903-35.

Kaczorek, T. (2013c). Zeroing of state variables in descriptor electrical circuits by state-feedbacks, Electrical Review 89(10): 200-203.

Kaczorek, T. (2012). Positive unstable electrical circuits, Electrical Review 88(5a): 187-192.

Kaczorek, T. (2011a). Positive electrical circuits and their reachability, Archives of Electrical Engineering 60(3): 283-301.

Kaczorek, T. (2011b). Positive systems consisting of $\mathrm{n}$ subsystems with different fractional orders, IEEE Transactions on Circuits and Systems 58(6): 1203-1210.

Kaczorek, T. (2011c). Selected Problems of Fractional Systems Theory, Springer, Berlin.

Kaczorek, T. (2010). Positive linear systems with different fractional orders, Bulletin of the Polish Academy of Sciences: Technical Sciences 58(3): 453-458.

Kaczorek, T. (2002). Positive $1 D$ and $2 D$ Systems, Springer, London.

Kaczorek, T. and Rogowski, K. (2015). Fractional Linear Systems and Electrical Circuits, Studies in Systems, Decision and Control, Vol. 13, Springer, Berlin.

Khalil R., Al Horani M., Yousef A., Sababheh M. (2014). A new definition of fractional derivative, Journal of Computational and Applied Mathematics 264: 65-70.

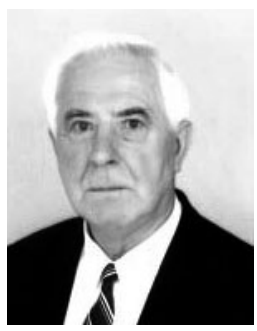

Tadeusz Kaczorek received his $\mathrm{MSc}, \mathrm{PhD}$ and DSc degrees in electrical engineering from the Warsaw University of Technology in 1956, 1962 and 1964, respectively. In the years 1968-1969 he was the dean of the Electrical Engineering Faculty, and in the period of 1970-1973 he was a deputy rector of the Warsaw University of Technology. In 1971 he became a professor and in 1974 a full professor at the same university. Since 2003 he has been a professor at the Białystok University of Technology. In 1986 he was elected a corresponding member and in 1996 a full member of the Polish Academy of Sciences. In the years 1988-1991 he was the director of the Research Center of the Polish Academy of Sciences in Rome. In 2004 he was elected an honorary member of the Hungarian Academy of Sciences. He has been granted honorary doctorates by 13 universities. His research interests cover systems theory, especially singular multidimensional systems, positive multidimensional systems, singular positive 1D and 2D systems, as well as positive fractional 1D and 2D systems. He initiated research in the field of singular 2D, positive 2D and positive fractional linear systems. $\mathrm{He}$ has published 28 books ( 8 in English) and over 1100 scientific papers. He has also supervised $69 \mathrm{PhD}$ theses. He is the editor-in-chief of the Bulletin of the Polish Academy of Sciences: Technical Sciences and a member of editorial boards of ten international journals.

Received: 24 July 2017

Revised: 30 October 2017 Accepted: 4 January 2018 